\title{
The relationship between the size of the seminal vesicles with premature ejaculation
}

\author{
Talal A. Abd-EIRaheem ${ }^{(1)}$, Noha E. Mohamed ${ }^{(1)}$, Ahmed AA Samak ${ }^{(2)}$ and \\ Mostafa M Samir ${ }^{(1)}$.
}

(1) Dermatology, STDs and andrology department, Faculty of Medicine

Fayoum University

(2)Radiology department, Faculty of Medicine, Zagazig University

Corresponding author: prof .Talal A. Abd-ElRaheem

E-mail address: talasam@yahoo.com

Tel:01006600360

Fax: +2 084636583

\begin{abstract}
Premature ejaculation (PE) is defined as short ejaculatory latency and lack of control upon ejaculation and described as one of the most common forms of sexual dysfunction in men. The ejaculatory progress is typically subdivided into three phases: emission, ejection, and orgasm. Emission consists of contractions of epididymis, vas deferens, seminal vesicles (SVs) and prostate, with expulsion of sperm and seminal fluid into the posterior urethra. The disorders of these organs may result in ejaculation disorder. Moreover, a higher frequency of sexual dysfunction was detected in patients with change in ultrasound in male accessory gland. Nowadays, many factors are considered to be related to SVs volume: Age \& sexual abstinence, prolactine level, inflammation, testosterone, thyroid hormones and smoking.However, data correlating PE with seminal vesicles $(\mathrm{SVs})$ are sparse. Transrectal ultrasound of seminal vesicles ( $\mathrm{SVs}$ ) should be considerd in the evaluation of patients with premature ejaculation. The aim of our study was to investigate whether a relationship exists between SVs volume and presence of PE. It was a crosssection, case-control study included 60
\end{abstract}

subjects divided into 2 groups. Patients group included $30 \mathrm{PE}$ patients 16 with acquired PE and 14 with lifelong PE, their ages ranged between 22-39 years with mean \pm SD $(30.57 \pm 4.77)$. Control group included 30 healthy matched subjects, their ages ranged between 2239 years with mean \pm SD $(30.23 \pm 4.61)$. The participants completed questionnaires concerning sexual health status including Premature Ejaculation Diagnostic Tool (PEDT), the international index of erectile function (IIEF). All patients and control cases underwent ultrasonography following 2 to 7 days of sexual abstinence. The prostate-vesicular region was assessed at rectal ultrasonography. This study demonstrated the relationship between the size of SV and PE. Men with a larger SV volume might have a higher PEDT score. Transrectal ultrasound of SV should be considered in the clinical evaluation of patients with PE.

Transrectal ultrasound of SV should be considered in the evaluation of patients with premature ejaculation.

KEYWORDS: Seminal Vesicles, Premature Ejaculation, Transrectal Ultrasound. 


\section{INTRODUCTION}

Premature ejaculation is defined as short ejaculatory latency and lack of control upon ejaculation and described as one of the most common forms of sexual dysfunction in men (Althof et al., 2010).

The ejaculatory progress is typically subdivided into three phases: emission, ejection (or penile expulsion), and orgasm (Rowland et al., 2010). Emission consists of contractions of epididymis, vas deferens, seminal vesicles (SVs) and prostate, with expulsion of sperm and seminal fluid into the posterior urethra. Ejection involves closure of bladder neck, pulsatile contractions of the bulbocavernosus and pelvic floor muscles, together with relaxation of the external urinary sphincter (La-Vignera et al., 2012).

The disorders of these organs may result in ejaculation disorder. Moreover, a higher frequency of sexual dysfunction was detected in male patients with change in ultrasound in male accessory gland (La Vignera et al., 2012).

Nowadays, many factors are considered to be related to SVs volume like, age \& sexual abstinence, abnormal prolactin level, inflammation, testosterone, thyroid hormones and smoking (Lotti et al., 2017).

However, data correlating PE with SVs are sparse. Transrectal ultrasound of seminal vesicle (SV) should be considered in the evaluation of patients with premature ejaculation (Hong et al., 2017).

\section{MATERIAL AND METHODS}

It was a cross-section, case-control study included 60 subjects divided into 2 groups. Patients group included $30 \mathrm{PE}$ patients 16 with acquired $\mathrm{PE}$ and 14 with lifelong PE, their ages ranged between 22-39 years with mean \pm SD (30.57 \pm 4.77$)$. Control group included 30 healthy matched subjects, their ages ranged between 22-39 years with mean $\pm \mathrm{SD}(30.23 \pm 4.61)$. The participants completed questionnaires concerning sexual health status including Premature Ejaculation Diagnostic Tool (PEDT), the international index of erectile function (IIEF). All patients and control cases underwent ultrasonography following 2 to 7 days of sexual abstinence. The prostate-vesicular region was assessed at rectal ultrasonography. The study plan was accepted by the Ethical committee of Faculty of Medicine, Fayoum University.

\section{Evaluations of the participants}

Inclusion and exclusion criteria:

- Age 20- 40 years (mean $\pm \mathrm{SD}$ ).

- In a heterosexual, stable, and monogamous sexual relationship with the same female partner for at least 6 months.

- They had erectile function domain of the International Index of Erectile Function-15 (IIEF-15) $\geq 26$, indicating normal erectile function.

- They were not consuming any drugs that affect sexual function or psychological status (e.g., selective serotonin reuptake inhibitors and phosphodiesterase type 5 inhibitors). - They were without any major psychiatric or somatic diseases.

- They did not have prostatitis-like symptoms such as complaints of perineal and/or ejaculatory pain or discomfort.

\section{$\underline{\text { Statistical analysis }}$}


Data were collected, revised, coded and entered to the Statistical Package for Social Science (IBM SPSS) version 23. The quantitative data were presented as mean, standard deviations and ranges when their distribution found parametric. Also qualitative variables were presented as number and percentages. The comparison between groups regarding qualitative data was done by using Chi-square test . The comparison between two independent groups with quantitative data and parametric distribution were done by using Independent t-test. The comparison between more than two

\section{RESULTS}

Regarding the total volume of seminal vesicles, there was a highly statistically significant difference, with p-value < 0.001 , in total volume of seminal vesicles in patients group than control group. There was a highly statistically significant negative correlation, with $\mathrm{p}$ value $<0.001$, regarding total volume of seminal vesicles on comparing with demographic data, total IIEF score and PEDT in control group. However, on comparing the correlation of total volume of seminal vesicles with demographic data, total IIEF score and PEDT in patients group, the relation of total volume of seminal vesicles with demographic data, PEDT classification and type of PE in patients group and

\section{DISCUSSION}

The aim of this study was to evaluate the relationship between the size of SV on ultrasound and PE. Thus, we tried to avoid other causes for PE. In this study, patients with PLS and infection of Chlamydia or Mycoplasma were excluded to avoid the impact of infection. Since transrectal ultrasound is a valuable diagnostic technique for evaluating indirect signs of ejaculatory independent groups with quantitative data and parametric distribution were done by using One Way Analysis of Variance (ANOVA).Spearman correlation coefficients were used to assess the correlation between two quantitative parameters in the same group. Receiver operating characteristic curve (ROC) were used to assess the best cut off point with its sensitivity, specificity, positive predictive value (PPV), negative predictive value (NPV) and area under curve (AUC). The confidence interval was set to $95 \%$ and the margin of error accepted was set to $5 \%$.

were not statistically significant. The relation of total IIEF score with demographic data, PEDT classification and type of PE in patients group was not statistically significant relation. On examining the correletion between control group and patients group regarding PEDT, there was a highly statistically significant increase, with $\mathrm{p}$ value $<0.001$, in PEDT in patients group than control group. This study demonstrated that the total volume of SVs in the patients with PE was significantly higher, with p-value < 0.01 , than that in the control cases, with higher incidence of areas of endocapsulation before ejaculation and of wall thickening and septa.

duct obstruction, such as dilatation, and the volume of all semen samples were more than $1.5 \mathrm{ml}$, we assumed that these patients did not have distal seminal tract obstruction. In addition, patients with other disorders that were associated with PE such as ED and hormonal disorders were excluded. There was no statistically significant difference regarding correletion between control 
group and patients group regarding demographic data of the studied cases. Comparison between control group and patients group regarding total volume of seminal vesicles showed that there was highly statistically significant increase in total volume of seminal vesicles in patients group than control group with p-value $<0.001$. Mereover, on comparing the correlation of total volume of seminal vesicles with demographic data, total IIEF score and PEDT in control group was highly statistically significant negative correlation between total volume of seminal vesicles and orgasmic function with $\mathrm{p}$-value $=0.006$ while no statistically significant correlation found between total volume of seminal vesicles and other parameters.

\section{CONCLUSION}

This study demonstrated the relationship between the size of SV and PE. Men with a larger total volume of SV might have a higher PEDT score. Transrectal ultrasound of SV should be considered in the clinical evaluation of patients with PE. However, the study simultaneously

\section{ACKNOWLEDGMENT:}

I am greatly thankful to Dr|Talal

Ahmed Abdel-Raheem, Professor of Dermatology, STDS and Andrology, Fayoum University, and Drl Noha Ezzat Mohammed, Lecturer of Dermatology

\section{REFERENCES:}

[1] La Vignera S, Condorelli R, Vicari E, D'Agata R, Calogero AE. (2012): High frequency of sexual dysfunction in patients with male accessory gland infections. Andrologia, 44, 438-46.

[2] Lotti F, Maseroli E, Fralassi N, Degl'Innocenti S, Boni L, et al.
Also, the relation of total IIEF score with demographic data and PEDT classification in control group showed that there was no statistically significant. On examining the correletion between control group and patients group regarding PEDT of the studied cases of this experiment showed highly statistically significant increase in PEDT in patients group than control group with $p$-value $<0.001$. These results demonstrated that patients with PE had larger mean APD of SV. We proposed that the enlargement of SV might be related to a stronger SV contraction with ejaculation, resulting in a more likely tendency to propel the seminal fluid into the posterior urethra, thus accelerating the phase of emission.

examined several end-points within the same population, allowing a valid comparison of the co-prevalence of the parameters examined, and supporting their possible association with a smoking habit.

STDS and Andrology, Fayoum University, for the precious advices, great help and support during the preparation of this work.

(2017). Is thyroid hormones evaluation of clinical value in the work-up of males of infertile couples? Hum Reprod; 31: 51829.

[3] Althof SE, Abdo CH, Dean J, Hackett G, McCabe M, McMahon CG, Rosen RC, 
Sadovsky R,Waldinger M, Becher E, Broderick GA, Buvat J, Goldstein I, EI-Meliegy AI, Giuliano F, Hellstrom WJ, Incrocci L, Jannini EA, Park K, Parish S, Porst H, Rowland D, Segraves R, Sharlip I, Simonelli C, Tan HM; (2010) International Society for Sexual Medicine. International Society For Sexual Medicine's guidelines for the diagnosis and treatment of premature ejaculation. J Sex Med;7:2947-69.
[4] Hong, Z , Feng Y, Ge Y , Jing J , Hu X, Shen J , Peng L , Yao B , Xin Z (2017). Relation of size of seminal vesicles on ultrasound to premature ejaculation, Asian Journal of Andrology 19, 554560.

[5] Rowland D, McMahon CG, Abdo C, Chen J, Jannini E, Waldinger MD, Ahn TY. (2010): Disorders of orgasm and ejaculation in men. J Sex Med, 7, 1668-86. 